\title{
Novel and recurrent PITX3 mutations in Belgian families with autosomal dominant congenital cataract and anterior segment dysgenesis have similar phenotypic and functional characteristics
}

Hannah Verdin ${ }^{1}$, Elena A Sorokina ${ }^{2}$, Françoise Meire ${ }^{3}$, Ingele Casteels ${ }^{4}$, Thomy de Ravel ${ }^{5}$, Elena V Semina ${ }^{2,6}$ and Elfride De Baere ${ }^{1 *}$

\begin{abstract}
Background: Congenital cataracts are clinically and genetically heterogeneous with more than 45 known loci and 38 identified genes. They can occur as isolated defects or in association with anterior segment developmental anomalies. One of the disease genes for congenital cataract with or without anterior segment dysgenesis (ASD) is PITX3, encoding a transcription factor with a crucial role in lens and anterior segment development. Only five unique PITX3 mutations have been described, of which the 17-bp duplication c.640_656dup, p.(Gly220Profs*95), is the most common one and the only one known to cause cataract with ASD. The aim of this study was to perform a genetic study of the PITX3 gene in five probands with autosomal dominant congenital cataract (ADCC) and ASD, to compare their clinical presentations to previously reported PITX3-associated phenotypes and to functionally evaluate the PITX3 mutations found.
\end{abstract}

Methods: Sanger sequencing of the coding region and targeted exons of PITX3 was performed in probands and family members respectively. Transactivation, DNA-binding and subcellular localization assays were performed for the PITX3 mutations found. Ophthalmological examinations included visual acuity measurement, slit-lamp biomicroscopy, tonometry and fundoscopy.

Results: In four Belgian families with ADCC and ASD the recurrent 17-bp duplication c.640_656dup, p.(Gly220Profs*95), was found in a heterozygous state. A novel PITX3 mutation c.573del, p.(Ser192Alafs*117), was identified in heterozygous state in a Belgo-Romanian family with a similar phenotype. Functional assays showed that this novel mutation retains its nuclear localization but results in decreased DNA-binding and transactivation activity, similar to the recurrent duplication.

Conclusions: Our study identified a second PITX3 mutation leading to congenital cataract with ASD. The similarity in phenotypic expression was substantiated by our in vitro functional studies which demonstrated comparable molecular consequences for the novel p.(Ser192Alafs*117) and the recurrent p.(Gly220Profs*95) mutations.

\footnotetext{
* Correspondence: elfride.debaere@ugent.be

${ }^{1}$ Center for Medical Genetics, Ghent University and Ghent University Hospital, Ghent, Belgium

Full list of author information is available at the end of the article
} 


\section{Background}

Cataract is defined as opacity or cloudiness of the crystalline lens. It is the primary cause of blindness worldwide and is classified into different types based on the age of onset. Congenital cataract manifests in the first year of life; the estimated incidence of congenital cataract is 72 per 100,000 children in developed countries with a higher incidence in less-developed countries. Congenital cataract is clinically and genetically heterogeneous with about 45 loci known and 38 genes identified. Mutations in genes encoding proteins important in the development and maintenance of the structural integrity of the lens such as crystallins, connexins and aquaporins [1,2] are typically associated with isolated congenital cataracts while mutations in the transcription factor genes PAX6 [3], FOXE3 [4], EYA1 [5], MAF [6], and PITX3 [7] have been described in congenital cataract with anterior segment dysgenesis (ASD). ASD is an umbrella term for the spectrum of developmental disorders affecting the structures of the anterior segment of the eye. The ocular anomalies typically include corneal opacity, adhesions between the iris and cornea or lens and cornea, iris hypoplasia, corectopia or polycoria, and malformation of the irido-corneal angle drainage structures [8].

PITX3 was isolated as the third gene of the PITX homeobox-containing transcription factor gene family [9,10], along with PITX1 [11,12] and PITX2 [13]. The PITX protein family is a subfamily of the paired-like class of the homeobox-containing proteins, which play a crucial role in the development of different organisms including mammals. Like the other members of this family, PITX3 contains a characteristic and strongly conserved homeodomain required for DNA binding. The conserved 14amino acid OAR motif, named after the homeodomain proteins otp, aristaless, and rax [14], is located downstream of this homeodomain, and may function in the target specificity and transactivation of the homeodomain protein $[13,14]$. Interestingly, Pitx3 mapped to the aphakia ( $a k$ ) locus [9], a recessive mutation resulting in bilateral microphthalmia with lens aplasia originally described by Varnum and Stevens in 1968. The lens develops normally in ak mice until an arrest occurs around embryonic days 10.5-11 [15] corresponding to the moment of initial expression of Pitx3 in the lens [7,9]. Consequently, Pitx3 was the top candidate for the $a k$ phenotype but no mutation was found in the coding region of Pitx3 [9]. Two different deletions in the promoter region of Pitx 3 were later found to explain the $a k$ phenotype $[16,17]$. Before this finding, the first human PITX3 mutations had already been identified in two families with autosomal dominant congenital cataract (ADCC). A 17-bp duplication c.640_656dup, p.(Gly220Profs*95), was found in a family with ADCC and ASD while a missense mutation c.38G > A, (Ser13Asn), was identified in a second family
[7]. Since the original gene identification study, there have been only a few additional mutations identified, bringing the total number of unique PITX3 mutations found in ADCC with or without ASD to five mutations in 13 different families [7,18-26]. Two of these mutations are recurrent, the most common one being the 17bp duplication p.(Gly220Profs*95) which was reported in eight of the 13 families [7,18-21,23,24]. The other recurrent mutation is a 1-bp deletion at position 650, p. (Gly217Alafs*92), found in two families [18,22]. Thus far, only the 17-bp duplication has been associated with ASD; all other mutations are reported with isolated cataract. Of particular note, homozygous PITX3 mutations were also described in two consanguineous pedigrees $[22,25]$.

The aim of this study was to analyze the PITX3 gene in five Belgian families with ADCC and ASD. We identified the recurrent 17-bp duplication c.640_656dup, p.(Gly220Profs*95), in four of these families, and a novel PITX3 mutation c.573del, p.(Ser192Alafs*117), in a fifth family. In vitro functional assays were performed for both mutations, showing similar functional characteristics. In conclusion, the similar ADCC and ASD phenotypes resulting from both mutations could be explained by our in vitro functional studies.

\section{Methods}

\section{Patients}

The consenting families enrolled in this study were referred for clinical genetic testing of ADCC and ASD. Prior to this study, they underwent mutation screening of the coding regions of the FOXC1 and PITX2 genes by Sanger sequencing and multiplex-ligation dependent probe amplification (MLPA), as described [27]. A total of four Belgian families and one Belgo-Romanian family were investigated for coding mutations in the PITX3 gene. Ophthalmological examinations included visual acuity measurements, slit-lamp biomicroscopy, tonometry and fundoscopy. The study was performed in accordance with the Declaration of Helsinki.

\section{Molecular genetic study of PITX3}

The coding region of PITX3 was screened using PCR amplification and subsequent Sanger sequencing. Primer pairs for the coding exons and PCR conditions can be found in Additional file 1. Sequencing was performed with the BigDye Terminator v3.1 Cycle Sequencing Kit on an ABI 3730XL genetic Analyzer, according to the manufacturer's instructions (Applied Biosystems, Carlsbad, CA). Mutation nomenclature is based on reference transcript NM_005029.3, with +1 corresponding to the A of the translation initiation codon ATG in the cDNA nomenclature, according to the Human Genome Variation Society (HGVS) nomenclature guidelines. 


\section{Functional characterization of PITX3 mutations DNA constructs, cell culture and luciferase assays}

The coding sequences corresponding to human PITX3 wild-type, the previously reported PITX3 p.(Gly220Profs"95) mutant [28] and the novel PITX3 p.(Ser192Alafs*117) mutant were cloned into the modified pcDNA3.1 expression vector (Invitrogen, Carlsbad, CA); all constructs were verified by DNA sequencing. The vector modification included an insertion of a C-terminal FLAG-tag followed by stop codon in frame with the PITX3 wild-type or mutant sequences. The PITX3 c.573del mutation was generated by QuickChange Lightning Site-Directed Mutagenesis kit (Stratagene, La Jolla, CA) according to the manufacturer's protocol.

Human lens epithelial cells (B3) (ATCC, Manassas, VA) were cultured in DMEM medium supplemented with 20\% fetal calf serum (FBS), glutamine, sodium pyruvate $(1 \mathrm{mM})$ and non-essential amino acids. The luciferase assays were performed with two different luciferase reporter vectors, $b c d$-TK-luc [28] and MIP656-bcd1,2-pGL3 [29] and as previously described. The experiments were performed two times in triplicate for both reporters. Student's paired t-test with a one-tailed distribution was utilized to determine the statistical significance of any differences in activity level.

\section{Immunocytochemistry}

B3 cells were cultured on coverslips to 50 to $80 \%$ confluency, fixed with $4 \%$ formaldehyde in PBS and then permeabilized with $0.25 \%$ Triton X-100. Indirect immunofluorescent staining was performed with monoclonal antiFLAG M2 antibody (Sigma, St. Louis, MO) and Alexa Fluor 568 donkey anti-mouse IgG (Invitrogen) as a secondary antibody. Hoechst 33342 (Invitrogen) was used as a nuclear counterstain.

\section{Electrophoretic mobility shift assay (EMSA)}

Nuclear extracts were prepared from B3 cells transiently transfected with the corresponding plasmids using the CelLytic NuCLEAR extraction kit (Sigma) as previously described [29]. In short, cells were harvested 48 hours after transfection and the cellular pellet was resuspended in hypotonic lysis buffer with protease inhibitor cocktail (Sigma). Igepal CA-630 was added after 15 minutes of incubation on ice, and then the cells were vortexed and spun down. Nuclear proteins were released by incubation of the crude nuclear pellet in the high-salt extraction buffer in the presence of protease inhibitors for 30 minutes on ice followed by centrifugation. Equal concentration of recombinant proteins was verified by Western blot densitometry (Image), NIH, Bethesda, MD). Oligonucleotide, 5' GATCCTAATCCCGTCGCGT CGTAATCCGGATC3', containing two bicoid sites separated by 10 nucleotides ( $B c d$ probe) was used in this study [28]. For oligonucleotide labeling and detection,
Biotin 3' End DNA Labeling Kit (Pierce, Rockford, IL) and LightShift Chemiluminescent EMSA Kit (Pierce) were used in accordance with the manufacturer's protocol. $20 \mathrm{fmol}$ of biotin end-labeled probe were mixed with binding buffer containing $50 \mathrm{ng} / \mu \mathrm{l}$ of Poly $(\mathrm{dI}-\mathrm{dC})$ and $2 \mu \mathrm{l}$ of nuclear extracts. After 20 minutes of incubation at room temperature, reactions were either diluted with $5 \times$ Loading buffer or further incubated for $30 \mathrm{mi}-$ nutes in presence of $1 \mu \mathrm{g}$ of goat polyclonal Pitx3 N-20 antibody (Santa Cruz Biotechnology, Dallas, TX) for the Supershift assay. Binding reactions and free probe were run on $5 \%$ native polyacrylamide gel in $0.5 \times \mathrm{TBE}$ buffer.

\section{Western blot}

For Western blot, equal volumes of whole cell extract from transfected cells were electrophoresed into 10\% SDS-polyacrylamide gel, transferred to polyvinylidine difluoride filters (Millipore, Billerica, MA) and probed with FLAG-M2 antibodies. After reaction with a secondary antibody conjugated with HRP, signal was detected with ECL Western Blotting Substrate (GE Healthcare, Little Chalfont, UK).

\section{Results \\ PITX3 mutations and associated phenotypes Recurrent PITX3 mutation}

In the index patients of four Belgian families, the recurrent duplication of 17-bp, c.640_656dup was found (Figure 1). This duplication creates a frameshift mutation, p.(Gly220Profs*95), leading to the replacement of the normal $82 \mathrm{C}$ terminal amino acids with 94 erroneous residues (Figure 2). Targeted mutation analysis in available family members demonstrated co-segregation of this mutation with disease (Figure 3).

The first Belgian family is a three-generation family with four affected individuals diagnosed with posterior subcapsular cataract (PSC) (Figure 3A). The recurrent duplication was found in the index patient (III:1), her brother (III:2) and her father (I:1).

The index patient (III:1) was born with severe corneal clouding in the right eye for which rotational corneal autografting was performed to obtain a clear optical axis, however this eye remained without functional visual acuity (VA). When she was 13 years old, she underwent lens extraction with lens implant of her left eye. Later on, she developed severe corneal endothelial cell loss in this eye and therefore underwent corneal transplant at the age of 15. A follow-up ophthalmological examination at the age of 26 revealed a VA for her right and left eye of light perception and 0.1, respectively, and corneal clouding with significant corneal endothelial loss. Her brother (III:2) also had congenital cataract with ASD. He was pseudophakic with a significant loss of endothelial cells. VA for his right and left eye was 0.5 and 0.6 , respectively. Their father 


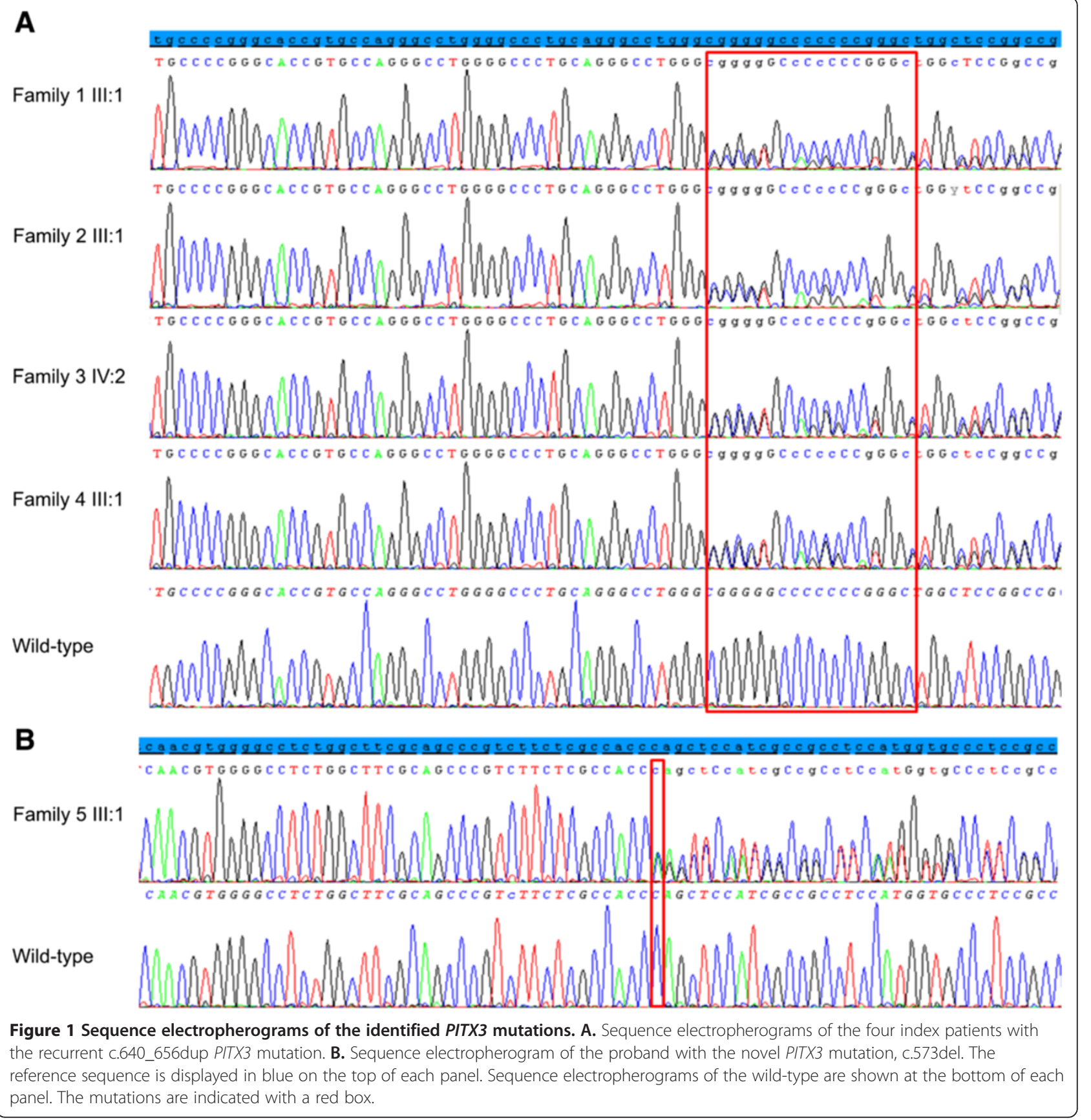

(II:1) has isolated bilateral congenital cataract. He underwent cataract extraction at the age of 18 . No other corneal or anterior segment anomalies were observed in him (Figure 4). All affected individuals (III:1, III:2 and II:2) have normal intraocular pressure (IOP).

In the second Belgian family there are three generations of affected individuals with ADCC (Figure 3B). The recurrent mutation c.640_656dup was found in the index case III:1; no DNA from other family members was available for targeted mutation testing. The cataracts of the index patient (III:1), her father (II:7), three paternal uncles (II:2, II:3, and II:4), and her paternal grandmother (I:2) developed gradually during childhood and became clinically apparent at different ages necessitating cataract extractions. Bilateral cataract extraction was performed in the index patient (III:1) at the age of 7 resulting in a bilateral best corrected visual acuity (BCVA) of 0.2. Apart from the cataract, the index patient has microcornea (bilateral corneal diameters of $9 \mathrm{~mm}$; microcornea is defined as a corneal diameter $<10 \mathrm{~mm}$ [30]), corneal opacities, iridocorneal adhesions, glaucoma and nystagmus (Figure 4). Over a period of three years she was 


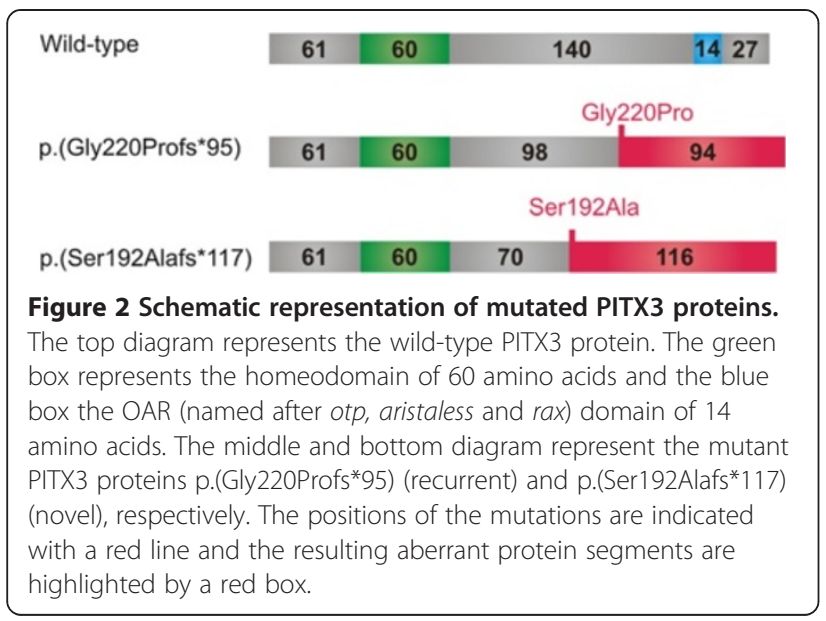

admitted twice for retinal detachment in her left eye. In addition, the index patient has intellectual disability. But as her mother and one maternal uncle (II:11) also display intellectual disability, this was felt to be unrelated to the paternally inherited PITX3 mutation.

The third Belgian family is a four-generation PSC family with nine affected individuals (Figure $3 C$ ). The recurrent duplication was found in the index patient (IV:2) and his mother (III:3). The index patient was diagnosed with bilateral congenital cataract and ASD. As severe central corneal clouding was present in his right eye, he underwent optical iridectomy however this eye remained amblyopic. Cataract extraction of his left eye was performed at the age of 8 . After the extraction, the patient's VA improved from 0.4 to 0.7 for his left eye while the VA for his right eye was light perception (Figure 4). His mother (III:3) presented only with bilateral congenital cataracts that were extracted at the age of 6 . Her affected brothers (III:4 and III:5) and mother (II:2) underwent cataract extraction at the age of 17,10 and 7 respectively.

The fourth Belgian family encompasses three generations of affected individuals with ADCC (Figure 3D). The recurrent duplication was found in the index case III:1; no DNA from other family members was available for targeted mutation testing. The index patient (III:1) is 3.5 years old and presented with nystagmus (manifesting latent left eye), corneal haze and iridocorneal adhesions with endothelial loss. The developmental anomaly was more severe in the right eye. VA for her right and left eye is light perception and 0.4, respectively. Her IOP was $12 \mathrm{mmHg}$ (Figure 4). Her father (II:6) only presented with congenital cataract that was extracted at the age of 12 .

\section{Novel PITX3 mutation}

In a Belgo-Romanian family with ADCC a novel 1-bp deletion c.573del was found in exon 4, p.(Ser192Alafs*117),

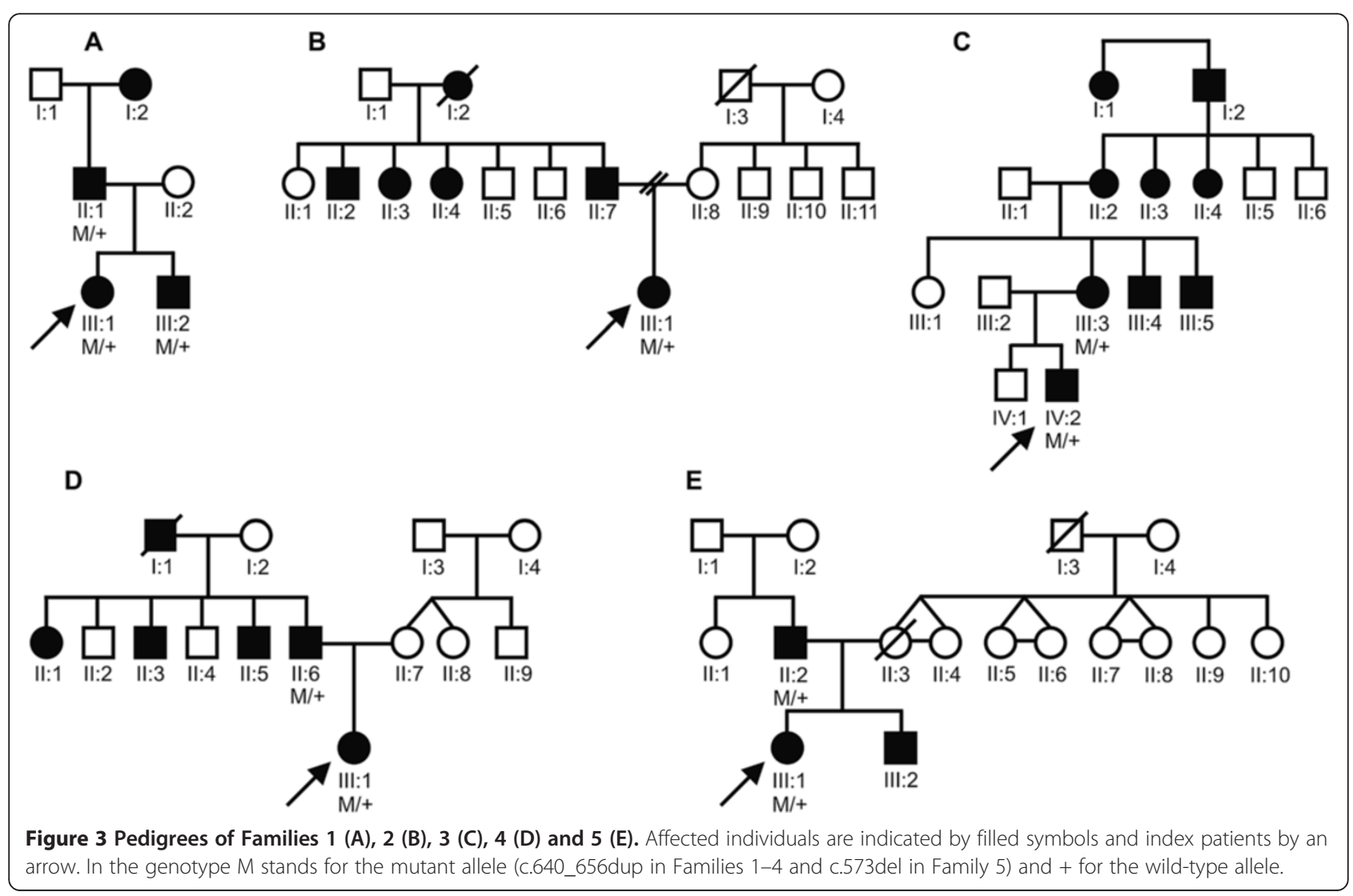




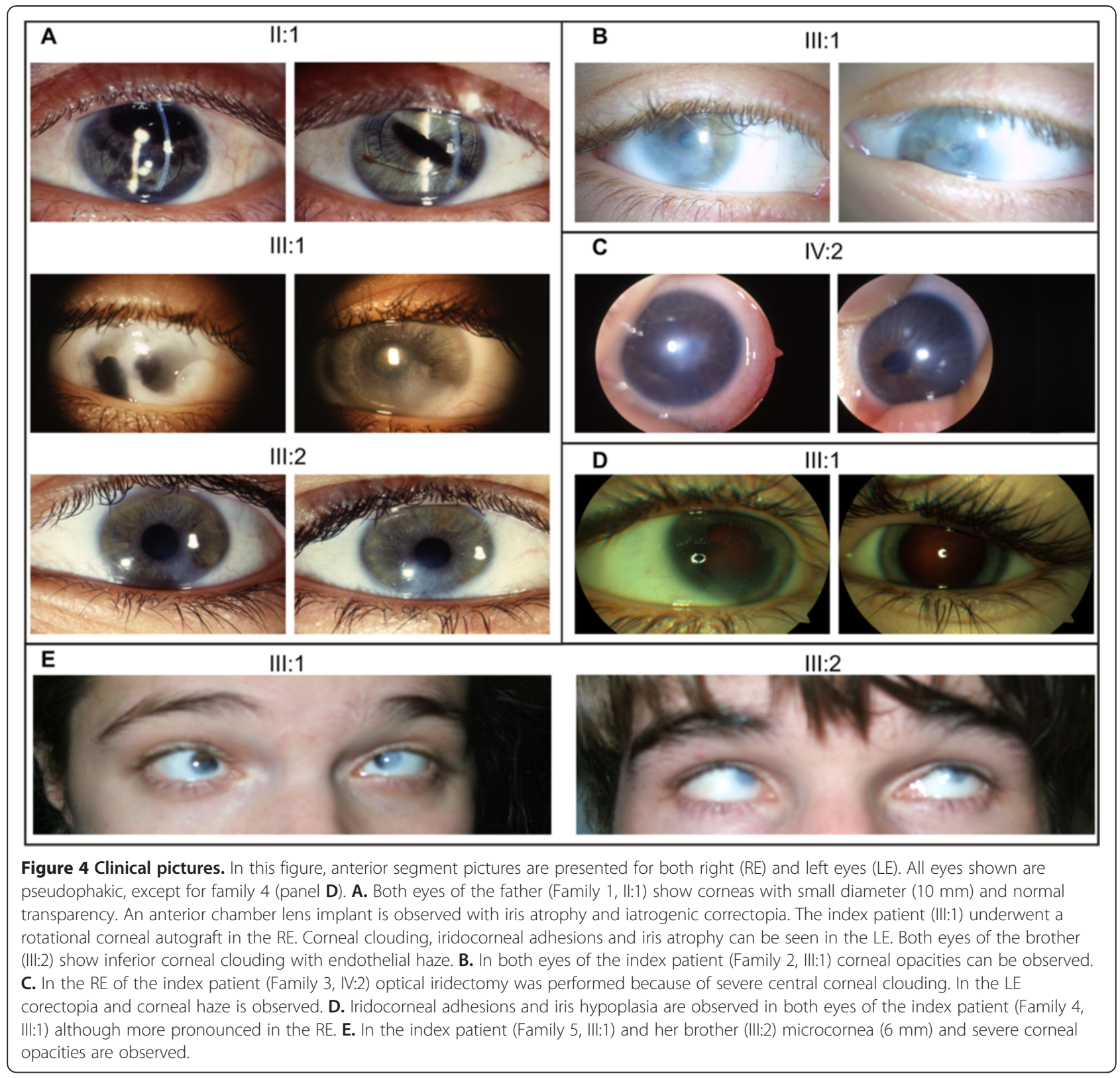

in the index patient (III:1) (Figure 1). Targeted testing identified this $1 \mathrm{bp}$-deletion in her father (II:2) as well; no DNA samples were available from other family members (Figure 3E). This 1-bp deletion creates a frameshift starting at codon 192 and introduces 116 novel residues before resulting in a stop codon downstream of the original stop codon (Figure 2). The index patient (III:1) was diagnosed with congenital cataract for which she underwent cataract extraction when she was 1 year old. Follow-up ophthalmological examination at the age of 25 demonstrated a VA for her right and left eye of light perception and 0.02, respectively, bilateral microcornea $(6 \mathrm{~mm})$, corneal opacities and iridocorneal adhesions. She underwent optical iridectomy of both eyes. Her IOP was normal. Her brother
(III:2) has microcornea $(5 \mathrm{~mm})$ and corneal opacities in addition to congenital cataract (Figure 4). Their father (II:2) has microcornea and congenital cataract.

\section{Functional characterization of novel PITX3 mutation} p.(Ser192Alafs*117) compared to the recurrent mutation p.(Gly220Profs*95)

\section{Subcellular localization}

Consistent with its role as a transcription factor, PITX3 was found to localize to the nucleus [28]. The novel PITX3 mutant p.(Ser192Alafs*117) displayed a similar nuclear localization as observed for the wild-type PITX3 and the previously reported recurrent mutant p.(Gly220Profs*95) (Figure 5A). 


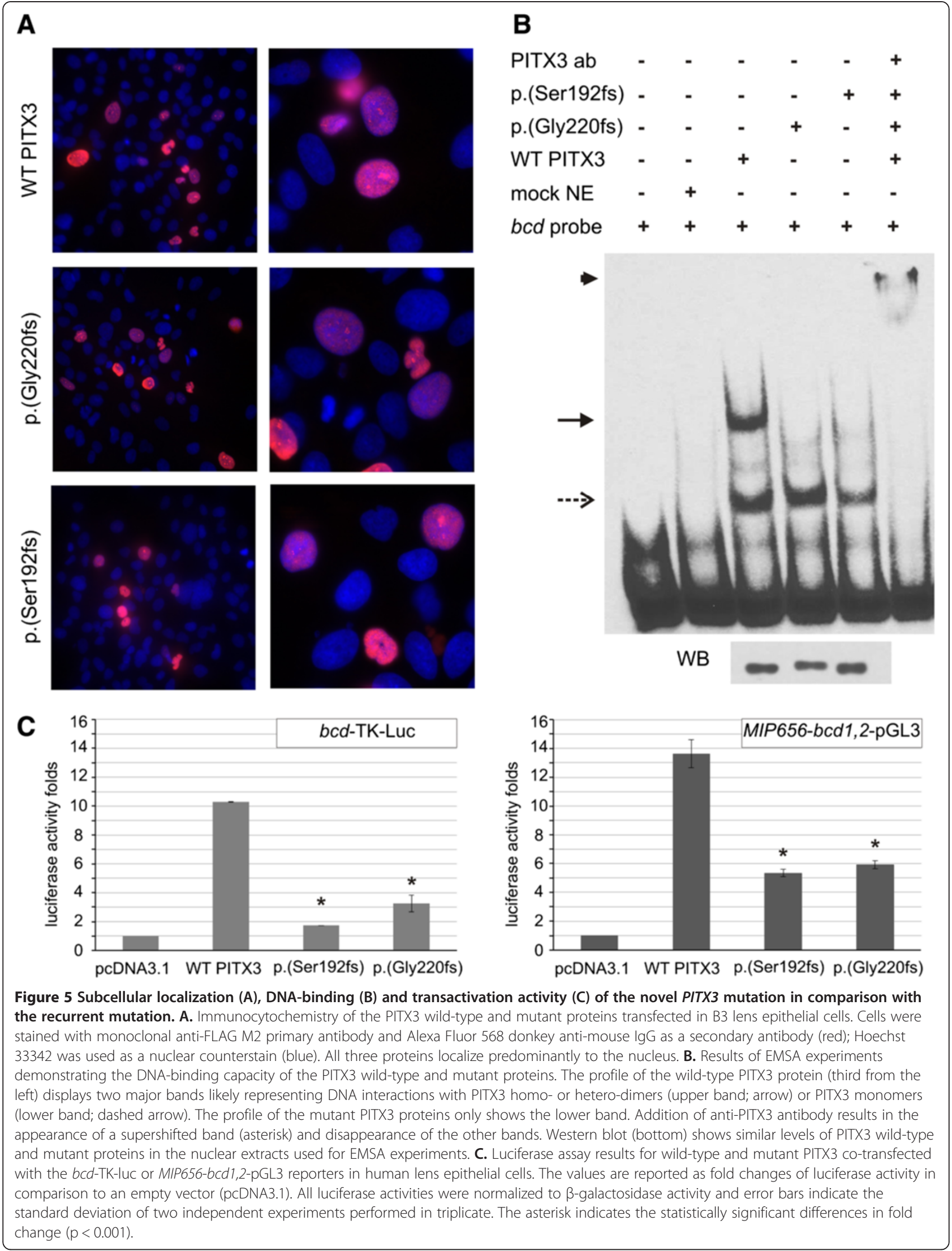




\section{Assessment of DNA-binding}

It has been previously shown that PITX3 mutations result in decreased DNA-binding activities [28]. To assess if the novel p.(Ser192Alafs*117) mutation has a similar effect on DNA-binding activity, we performed EMSA for the novel mutant in comparison to the previously reported recurrent p.(Gly220Profs*95) mutant and wild-type proteins. The results were also compared to the EMSA profiles of the $b c d$ probe alone and with mock nuclear extracts (NE) to exclude nonspecific binding activity. The observed profile for the wild-type protein consisted of two major bands in agreement with previously reported results (Figure 5B). The fast-migrating band probably corresponds to PITX3 binding DNA as a monomer and the slower migrating bands may represent PITX3 homodimer- or PITX3 heterodimer-DNA complexes with other proteins [13,28]. The slower migrating bands have disappeared in the EMSA profiles of both PITX3 mutants while the fastmigrating band, likely representing monomeric DNAbinding activity, remained. The specificity of the assay was verified by adding an anti-PITX3 antibody resulting in a supershifted band and fading of the other bands (Figure 5B). Western blot analyses were performed to confirm the equal presence of wild-type and mutant PITX3 protein in the nuclear extract used for the EMSA experiments (Figure 5B).

\section{Transactivation activity}

In addition to altered DNA-binding properties, it has also been shown that PITX3 mutants exhibit a reduced transactivation activity [28]. Using luciferase assays in human lens epithelial cells we evaluated the transactivation activity of the novel PITX3 mutant p.(Ser192Alafs"117) in comparison to the recurrent p.(Gly220Profs*95) mutant and wild-type proteins (Figure 5C). Co-transfection of the wild-type PITX3 with the $b c d$-TK-luc reporter resulted in a 10-fold increase in luciferase activity in comparison with an empty pcDNA3.1 vector, while the novel p. (Ser192Alafs"117) and recurrent p.(Gly220Profs"95) mutants resulted in a $\sim 1.7$-fold (16.8\% of wild-type activity; $\mathrm{p}<0.001$ ) and $\sim 3.2$ fold increase (31.5\% of wild-type activity; $\mathrm{p}<0.001$ ), respectively. A similar trend could be observed using the MIP656- $b c d 1,2-$ luc reporter, as a 13fold increase in luciferase activity was detected when this reporter was co-transfected with wild-type PITX3. In contrast, the luciferase activity for the novel and recurrent PITX3 mutants was found to be $39.2 \%$ and $43.4 \%$ $(\mathrm{p}<0.001)$ of wild-type activity, respectively.

\section{Discussion}

Congenital cataract is a clinically and genetically heterogeneous condition. Most genes are associated with isolated congenital cataracts while mutations in the transcription factor genes PAX6, FOXE3, EYA1, MAF, and PITX3 lead to congenital cataract with ASD [3-7]. Of particular note, PITX3 mutations can cause isolated congenital cataract as well as ASD-associated cataract, even within the same family [7]. This inter- and intrafamilial phenotypic variability can likely be ascribed to modifier genes or environmental factors. PITX3 mutations represent a rare cause of congenital cataract with or without ASD. Indeed, only five unique PITX3 mutations in 13 different families were known before the onset of this study (Table 1 ). The most common mutation is the 17-bp duplication c.640_656dup accounting for more than half of the families $[7,18-21,23,24]$ and ADCC with ASD has been documented only in association with this duplication. Notably, the novel PITX3 mutation c.573del, p.(Ser192Alafs"117), was found in a family with $\mathrm{ADCC}$ and ASD, thus being the second mutation leading to this phenotype. Like the other reported PITX3 mutations, this novel frameshift mutation is located outside the homeodomain. Interestingly, all PITX3 frameshift mutants lack the C-terminal 14-amino-acid motif (see Additional file 2), which is conserved in numerous paired-like homeodomain proteins and is named the OAR domain, after the homeodomain genes otp, aristaless, and rax [14]. The function of this OAR domain is not fully established yet but it has been suggested that it could interact with additional proteins, hence mediating the target specificity of the homeobox-containing transcription factors $[13,14]$. Although the OAR domain is conserved in all of these homeodomain proteins, its function appears to vary among the proteins. Functional assays with different deletion mutants of Otp showed that mutants missing the region downstream of the homeodomain, including the OAR domain, presented with a different DNA-binding profile and a substantial reduction of transactivation activity, thus suggesting an activating regulatory role [31]. The OAR domain is also important for the transactivation activity of SHOX as a truncated SHOX mutant lacking this domain has an abolished transactivation activity [32]. In contrast, a study of the Pitx2 C-terminus demonstrates an inhibitory role for the OAR domain as it was shown that the presence of this domain inhibits DNA-binding while masking this domain through protein interaction stimulates DNA-binding and transcriptional activation [33]. This finding was supported by studies in Prx2 and Cart1 where the OAR domain inhibits transactivation and serves as an intramolecular switch of its transactivation activity, respectively $[34,35]$. To explore the function of the Cterminal region in PITX3, Sakazume et al. investigated the effect of PITX3 mutants on DNA-binding and transactivation activity. In accordance with the Otp and SHOX study, an altered DNA-binding profile and diminished transcriptional activity was shown [28].

Both the novel and recurrent PITX3 mutations found here disrupt the C-terminus including the OAR domain. As they both lead to a similar phenotype, we assessed 
Table 1 Overview of the different PITX3 mutations described in human and associated phenotypic details

\begin{tabular}{|c|c|c|c|}
\hline Mutation & Mode of inheritance & Clinical features & Reference \\
\hline c.38G > A, p.(Ser13Asn) & Dominant & $\begin{array}{l}\text { Mother and son with ADCC and glaucoma at a } \\
\text { later age. }\end{array}$ & 7 \\
\hline c.542del, p.(Pro181Leufs*128) & Dominant & Four-generation English family with isolated PPC. & 26 \\
\hline c.573del, p.(Ser192Alafs*117) & Dominant & $\begin{array}{l}\text { Belgo-Romanian family with ADCC. Two individuals } \\
\text { also have ASD. }\end{array}$ & This study, Family 5 \\
\hline \multirow[t]{12}{*}{ c.640_656dup, p.(Gly220Profs*95) } & \multirow[t]{12}{*}{ Dominant } & Six-generation family with ADCC and ASD. & 7 \\
\hline & & $\begin{array}{l}\text { Four-generation English family with PPC. One } \\
\text { individual also has ASD and congenital glaucoma. }\end{array}$ & 18,19 \\
\hline & & $\begin{array}{l}\text { Four-generation English family with PPC. Four } \\
\text { individuals also have ASD. }\end{array}$ & 18,19 \\
\hline & & Five-generation English family with isolated PPC. & 18,19 \\
\hline & & Four-generation Chinese family with isolated PPC. & 18 \\
\hline & & $\begin{array}{l}\text { Four-generation British/German family with isolated } \\
\text { PPC. }\end{array}$ & 20 \\
\hline & & Five-generation English family with isolated PPC. & 21 \\
\hline & & $\begin{array}{l}\text { Four-generation Australian family with PSC. Seven } \\
\text { individuals also have ASD. }\end{array}$ & 23,24 \\
\hline & & $\begin{array}{l}\text { Three-generation Belgian family with PSC. Two } \\
\text { individuals also have ASD. }\end{array}$ & This study, Family 1 \\
\hline & & $\begin{array}{l}\text { Three-generation Belgian family with ADCC. One } \\
\text { individual also has ASD. }\end{array}$ & This study, Family 2 \\
\hline & & $\begin{array}{l}\text { Four-generation Belgian family with PSC. One } \\
\text { individual also has ASD. }\end{array}$ & This study, Family 3 \\
\hline & & $\begin{array}{l}\text { Three-generation Belgian family with ADCC. One } \\
\text { individual also has ASD. }\end{array}$ & This study, Family 4 \\
\hline c.640_656del, p.(Ala214Argfs*42) & Recessive & $\begin{array}{l}\text { Daughter from healthy first cousin parents with } \\
\text { ASD and severe congenital microphthalmia. }\end{array}$ & 25 \\
\hline \multirow[t]{2}{*}{ c.650del, p.(Gly217Alafs*92) } & Dominant & $\begin{array}{l}\text { Four-generation Hispanic family with isolated } \\
\text { cataract. }\end{array}$ & 18 \\
\hline & Dominant/recessive & $\begin{array}{l}\text { Three-generation Lebanese family with PPC. Two } \\
\text { brothers from a consanguineous mating showed a } \\
\text { more severe ocular and neurologic phenotype in } \\
\text { addition to PPC. }\end{array}$ & 22 \\
\hline $\begin{array}{l}\text { Microdeletion of 10q24.32 } \\
\text { encompassing PITX3 }\end{array}$ & De novo & $\begin{array}{l}\text { Patient with Smith-Magenis syndrome-like behav- } \\
\text { ioural abnormalities, intellectual disability and dys- } \\
\text { morphic features but no eye phenotype. }\end{array}$ & 41 \\
\hline
\end{tabular}

Mutation nomenclature is based on reference transcript NM_005029.3 and the HGVS guidelines.

ADCC: autosomal dominant congenital cataract; PPC: posterior polar cataract; ASD: anterior segment dysgenesis; PSC: posterior subcapsular cataract.

whether this similarity could be substantiated by in vitro functional assays. Since all PITX3 frameshift mutations utilize a stop codon located downstream of the normal stop codon in the last coding exon, they are not predicted to be subject to nonsense mediated decay (NMD). The novel PITX3 mutant retained its nuclear localization but displayed altered DNA-binding properties similar to the recurrent PITX3 p.(Gly220Profs*95) mutant. In addition, a similar decrease of transcriptional activity compared to wild-type PITX3 was observed for the novel and recurrent PITX3 mutants. These mutations may produce aberrant protein that can either antagonize DNA-binding activity of wild-type proteins or form inactive dimers with wild- type proteins, impairing wild-type protein function in both situations. Co-transfection assays with mutant and wild-type PITX3 could not, however, substantiate a dominant-negative effect [28]. A loss-of-function effect for the PITX3 mutations is therefore more probable. This view is supported by the report on a large ADCC pedigree in which two brothers from a consanguineous mating are homozygous for the PITX3 mutation segregating in the family and manifest a more severe ocular and neurological phenotype, with severe microphthalmia and neurological involvement in addition to cataract [22]. This severe combination of ocular and neurological involvement has also been described in the two spontaneous 
Pitx3 mutant mice, aphakia and eyeless [9,10,15,36]. Interestingly, besides its expression in the developing lens, Pitx3 expression was also observed in the dopamine neurons of the midbrain [10]. A severe loss of dopaminergic neurons in the substantia nigra was also observed in aphakia and eyeless mice. As these neurons play important roles in the control of movement, emotion, cognition, and reward related behavior, this neuronal loss can explain the observed locomotor and behavioural defects [36-39]. Unlike the human PITX3 mutations, heterozygous Pitx3 mice were phenotypically normal which might be attributed to interspecies differences, as mice seem to be less sensitive to gene dosage. However, the loss-of-function hypothesis is challenged by the report on another consanguineous mating between phenotypically normal first cousins resulted in a girl homozygous for the PITX3 mutation c.640_656del, manifesting as severe bilateral microphthalmia and ASD but without cataract or a neurological phenotype [25]. This phenotype is reminiscent of the manifestations in the Texel Sheep as this breed displays microphthalmia as an autosomal recessive congenital condition, caused by a missense mutation in the conserved homeodomain of PITX3, c.338G > C, p.(Arg113Pro) [40]. In addition, a heterozygous microdeletion of 10q24.32 encompassing PITX3 in a patient with Smith-Magenis syndrome-like behavioral abnormalities was recently described. The patient also presented with intellectual disability and dysmorphic features but, surprisingly, lacked an eye phenotype. Analysis of neurotransmitters in his cerebrospinal fluid revealed an absence of L-DOPA and LDOPA treatment led to mild improvement of his behavior [41]. Based on these observations and the selective loss of dopaminergic neurons in the Pitx3 mutant mice, it might be interesting to assess the dopaminergic function of patients with PITX3 mutations.

Taken together, these findings suggest that PITX3 associated phenotypes most likely result from a more complex mutational mechanisms than loss of function effects alone and that more extensive studies are needed to correlate the phenotypic and molecular consequences of PITX3 mutations.

\section{Conclusions}

In this study, we identified a novel PITX3 mutation, p. (Ser192Alafs"117) and the recurrent p.(Gly220Profs"95) mutation in one Belgo-Romanian family and four Belgian families with ADCC and ASD, respectively. The novel mutation p.(Ser192Alafs"117) is only the second PITX3 mutation to be associated with this combined phenotype, along with the recurrent mutation p.(Gly220Profs"95). The similarity in phenotypic expression could be explained by in vitro functional studies which demonstrated similar molecular consequences for the novel p.(Ser192Alafs"117) and the recurrent p.(Gly220Profs"95) mutations.

\section{Additional files}

\section{Additional file 1: PCR primers and conditions for molecular screening of PITX3.}

Additional file 2: Schematic representation of all reported mutated PITX3 proteins. The top diagram represents the wild-type PITX3 protein. The green box displays the homeodomain of 60 amino acids and the OAR (named after otp, aristaless and rax) domain of 14 amino acids is displayed by a blue box. The recurrent p.(Gly220Profs*95) and novel p.(Ser192Alafs*117) mutations are indicated in bold. The positions of the mutations are indicated with a red line and a red box displays the resulting aberrant protein segments.

\section{Competing interests}

The authors declare that they have no competing interests.

\section{Authors' contributions}

HV carried out the molecular genetic studies and drafted the manuscript. EAS performed the immunocytochemistry, EMSA experiments and luciferase assays. FM, TDR and IC performed the ophthalmological examinations and provided clinical information. EVS and EDB participated in the design and coordination of the study, and helped to draft the manuscript. All authors read and approved the final manuscript.

\section{Acknowledgements}

We wish to thank Sally Hooghe for her expert technical assistance, Linda Reis for careful reading of the manuscript and helpful comments, and Patricia Delbeke for useful clinical advice. We are most grateful to the families who participated in this study. HV was a doctoral fellow from the Research Foundation Flanders (FWO) from 2009-2013. EDB is a senior clinical investigator from the FWO. This study is supported by FWO grant G079711N. EVS is supported by $\mathrm{NIH}$ award EY015518.

\section{Author details}

${ }^{1}$ Center for Medical Genetics, Ghent University and Ghent University Hospital, Ghent, Belgium. ²Department of Pediatrics and Children's Research Institute, Medical College of Wisconsin, Milwaukee 53226, WI, USA. ${ }^{3}$ Department of Paediatric Ophthalmology, Queen Fabiola Children's University Hospital, Brussels, Belgium. ${ }^{4}$ Department of Ophthalmology, Leuven University Hospitals, Leuven, Belgium. ${ }^{5}$ Center for Human Genetics, Leuven University Hospitals, Leuven, Belgium. ${ }^{6}$ Department of Cell Biology, Neurobiology and Anatomy, Medical College of Wisconsin, Milwaukee 53226, WI, USA.

Received: 1 December 2013 Accepted: 10 February 2014 Published: 20 February 2014

\section{References}

1. Graw JJ: Congenital hereditary cataracts. Int J Dev Biol 2003, 48:1031-1044.

2. Shiels A, Hejtmancik JF: Genetics of human cataract. Clin Genet 2013, 84:120-127.

3. Hanson I, Churchill A, Love J, Axton R, Moore T, Clarke M, Meire F, Van Heyningen $\mathrm{V}$ : Missense mutations in the most ancient residues of the PAX6 paired domain underlie a spectrum of human congenital eye malformations. Hum Mol Genet 1999, 8:165-172.

4. Semina EV, Brownell I, Mintz-Hittner HA, Murray JC, Jamrich M: Mutations in the human forkhead transcription factor FOXE3 associated with anterior segment ocular dysgenesis and cataracts. Hum Mol Genet 2001, 10:231-236.

5. Azuma NN, Hirakiyama AA, Inoue $\Pi$, Asaka AA, Yamada MM: Mutations of a human homologue of the Drosophila eyes absent gene (EYA1) detected in patients with congenital cataracts and ocular anterior segment anomalies. Hum Mol Genet 2000, 9:363-366.

6. Jamieson RVR, Perveen RR, Kerr BB, Carette MM, Yardley JJ, Heon EE, Wirth MGM, Van Heyningen W, Donnai DD, Munier FF, Black GCMG: Domain disruption and mutation of the bZIP transcription factor, MAF, associated with cataract, ocular anterior segment dysgenesis and coloboma. Hum Mol Genet 2001, 11:33-42.

7. Semina EV, Ferrell RE, Mintz-Hittner HA, Bitoun P, Alward WLM, Reiter RS, Funkhauser C, Daack-Hirsch S, Murray JC: A novel homeobox gene PITX3 is mutated in families with autosomal-dominant cataracts and ASMD. Nat Genet 1998, 19:167-170. 
8. Idrees F, Vaideanu D, Fraser SG, Sowden JC, Khaw PT: A review of anterior segment dysgeneses. Surv Ophthalmol 2006, 51:213-231.

9. Semina EV, Reiter RS, Murray JC: Isolation of a new homeobox gene belonging to the Pitx/Rieg family: expression during lens development and mapping to the aphakia region on mouse chromosome 19. Hum Mol Genet 1997, 6:2109-2116.

10. Smidt MP, Van Schaick HS, Lanctôt C, Tremblay JJ, Cox JJ, van der Kleij AA, Wolterink G, Drouin J, Burbach JP: A homeodomain gene Ptx3 has highly restricted brain expression in mesencephalic dopaminergic neurons. Proc Natl Acad Sci USA 1997, 94:13305-13310.

11. Lamonerie TT, Tremblay JJ, Lanctôt CC, Therrien MM, Gauthier Y, Drouin J: Ptx1, a bicoid-related homeo box transcription factor involved in transcription of the pro-opiomelanocortin gene. Genes Dev 1996, 10:1284-1295.

12. Szeto DPD, Ryan AKA, O'Connell SMS, Rosenfeld MGM: P-OTX: a PIT-1interacting homeodomain factor expressed during anterior pituitary gland development. Proc Natl Acad Sci USA 1996, 93:7706-7710.

13. Semina EV, Reiter R, Leysens NJ, Alward WLM, Small KW, Datson NA Siegel-Bartelt J, Bierke-Nelson D, Bitoun P, Zabel BU, Carey JC, Murray JC: Cloning and characterization of a novel bicoid-related homeobox transcription factor gene, RIEG, involved in Rieger syndrome. Nat Genet 1996, 14:392-399.

14. Furukawa $\Pi$, Kozak CAC, Cepko CL: rax, a novel paired-type homeobox gene, shows expression in the anterior neural fold and developing retina. Proc Natl Acad Sci USA 1997, 94:3088-3093.

15. Varnum DS, Stevens LC: Aphakia, a new mutation in the mouse. $J$ Hered 1968, 59:147-150.

16. Semina EV, Murray JC, Reiter R, Hrstka RF, Graw J: Deletion in the promoter region and altered expression of Pitx3 homeobox gene in aphakia mice. Hum Mol Genet 2000, 9:1575-1585.

17. Rieger DK, Reichenberger E, McLean W, Sidow A, Olsen BR: A doubledeletion mutation in the Pitx3 gene causes arrested lens development in aphakia mice. Genomics 2001, 72:61-72.

18. Berry V, Yang Z, Addison PKF, Francis PJ, Ionides A, Karan G, Jiang L, Lin W, Hu J, Yang R, Moore A, Zhang K, Bhattacharya SS: Recurrent 17 bp duplication in PITX3 is primarily associated with posterior polar cataract (CPP4). J Med Genet 2004, 41:e109.

19. Addison PKF, Berry V, lonides ACW, Francis PJ, Bhattacharya SS, Moore AT: Posterior polar cataract is the predominant consequence of a recurrent mutation in the PITX3 gene. Br J Ophthalmol 2005, 89:138-141.

20. Finzi S, Li Y, Mitchell TN, Farr A, Maumenee IH, Sallum JMF, Sundin O: Posterior polar cataract: genetic analysis of a large family. Ophthalmic Genet 2005, 26:125-130

21. Burdon KP, McKay JD, Wirth MG, Russell-Eggit IM, Bhatti S, Ruddle JB, Dimasi DP, Mackey DA, Craig JE: The PITX3 gene in posterior polar congenital cataract in Australia. Mol Vis 2006, 12:367-371.

22. Bidinost C, Matsumoto M, Chung D, Salem N, Zhang K, Stockton DW, Khoury A, Megarbane A, Bejjani BA, Traboulsi El: Heterozygous and homozygous mutations in PITX3 in a large Lebanese family with posterior polar cataracts and neurodevelopmental abnormalities. Invest Ophthalmol Vis Sci 2006, 47:1274-1280.

23. Summers KM, Withers SJ, Gole GA, Piras S, Taylor PJ: Anterior segment mesenchymal dysgenesis in a large Australian family is associated with the recurrent 17 bp duplication in PITX3. Mol Vis 2008, 14:2010-2015.

24. Withers SJ, Gole GA, Summers KM: Autosomal dominant cataracts and Peters anomaly in a large Australian family. Clin Genet 1999, 55:240-247.

25. Aldahmesh MA, Khan AO, Mohamed J, Alkuraya FS: Novel recessive BFSP2 and PITX3 mutations: insights into mutational mechanisms from consanguineous populations. Genet Med 2011, 13:978-981.

26. Berry V, Francis PJ, Prescott Q, Waseem NH, Moore AT, Bhattacharya SS: A novel 1-bp deletion in PITX3 causing congenital posterior polar cataract. Mol Vis 2011, 17:1249-1253.

27. D'haene B, Meire F, Claerhout I, Kroes HY, Plomp A, Arens YH, De Ravel T, Casteels I, De Jaegere S, Hooghe S, Wuyts W, van den Ende J, Roulez F, Veenstra-Knol HE, Oldenburg RA, Giltay J, Verheij JBGM, De Faber J-T, Menten B, De Paepe A, Kestelyn P, Leroy BP, De Baere E: Expanding the spectrum of FOXC1 and PITX2 mutations and copy number changes in patients with anterior segment malformations. Invest Ophthalmol Vis Sci 2011, 52:324-333.

28. Sakazume S, Sorokina E, Iwamoto $Y$, Semina EV: Functional analysis of human mutations in homeodomain transcription factor PITX3. BMC MO Biol 2007, 8:84
29. Sorokina EA, Muheisen S, Mlodik N, Semina EV: MIP/Aquaporin 0 represents a direct transcriptional target of PITX3 in the developing lens. PLOS ONE 2011, 6:e21122

30. Traboulsi El: Genetic diseases of the eye. Oxford: Oxford University Press; 2011.

31. Simeone A, D'Apice MR, Nigro V, Casanova J, Graziani F, Acampora D, Avantaggiato $\mathrm{V}$ : Orthopedia, a novel homeobox-containing gene expressed in the developing CNS of both mouse and Drosophila. Neuron 1994, 13:83-101.

32. Rao E, Blaschke RJ, Marchini A, Niesler B, Burnett M, Rappold GA: The LeriWeill and Turner syndrome homeobox gene SHOX encodes a cell-type specific transcriptional activator. Hum Mol Genet 2001, 10:3083-3091.

33. Amendt BA, Sutherland LB, Russo AF: Multifunctional role of the Pitx2 homeodomain protein C-terminal tail. Mol Cell Biol 1999, 19:7001-7010

34. Norris RA, Kern MJ: Identification of domains mediating transcription activation, repression, and inhibition in the paired-related homeobox protein, Prx2 (S8). DNA Cell Biol 2001, 20:89-99.

35. Brouwer A, Berge Ten D, Wiegerinck R, Meijlink F: The OAR/aristaless domain of the homeodomain protein Cart1 has an attenuating role in vivo. Mech Dev 2003, 120:241-252.

36. Rosemann M, Ivashkevich A, Favor J, Dalke C, Hölter SM, Becker L, Rácz I, Bolle I, Klempt M, Rathkolb B, Kalaydjiev S, Adler T, Aguilar A, Hans W, Horsch M, Rozman J, Calzada-Wack J, Kunder S, Naton B, Gailus-Durner V Fuchs H, Schulz H, Beckers J, Busch DH, Burbach JPH, Smidt MP, Quintanilla-Martinez L, Esposito I, Klopstock T, Klingenspor M, et al: Microphthalmia, parkinsonism, and enhanced nociception in Pitx3 (416insG ) mice. Mamm Genome 2010, 21:13-27.

37. van den Munckhof P, Luk KC, Ste-Marie L, Montgomery J, Blanchet PJ, Sadikot AF, Drouin J: Pitx3 is required for motor activity and for survival of a subset of midbrain dopaminergic neurons. Development 2003, 130:2535-2542.

38. Ardayfio P, Moon J, Leung KKA, Youn-Hwang D, Kim K-S: Impaired learning and memory in Pitx3 deficient aphakia mice: a genetic model for striatum-dependent cognitive symptoms in Parkinson's disease. Neurobiol Dis 2008, 31:406-412.

39. Hwang D-Y, Ardayfio P, Kang UJ, Semina EV, Kim K-S: Selective loss of dopaminergic neurons in the substantia nigra of Pitx3-deficient aphakia mice. Brain Res Mol Brain Res 2003, 114:123-131.

40. Becker D, Tetens J, Brunner A, Bürstel D, Ganter M, Kijas J, International Sheep Genomics Consortium, Drögemüller C: Microphthalmia in Texel sheep is associated with a missense mutation in the paired-like homeodomain 3 (PITX3) gene. PLOS ONE 2010, 5:e8689.

41. Derwińska K, Mierzewska H, Goszczańska A, Szczepanik E, Xia Z, Kuśmierska K, Tryfon J, Kutkowska-Kaźmierczak A, Bocian E, Mazurczak T, Obersztyn E, Stankiewicz P: Clinical improvement of the aggressive neurobehavioral phenotype in a patient with a deletion of PITX3 and the absence of L-DOPA in the cerebrospinal fluid. Am J Med Genet B Neuropsychiatr Genet 2012, 159B:236-242.

doi:10.1186/1750-1172-9-26

Cite this article as: Verdin et al:: Novel and recurrent PITX3 mutations in Belgian families with autosomal dominant congenital cataract and anterior segment dysgenesis have similar phenotypic and functional characteristics. Orphanet Journal of Rare Diseases 2014 9:26.

\section{Submit your next manuscript to BioMed Central and take full advantage of:}

- Convenient online submission

- Thorough peer review

- No space constraints or color figure charges

- Immediate publication on acceptance

- Inclusion in PubMed, CAS, Scopus and Google Scholar

- Research which is freely available for redistribution 